\section{El análisis estratigráfico constructivo y el proyecto de restauración arquitectónica}

\author{
Camilla Mileto, Fernando Vegas \\ Universidad Politécnica de Valencia
}

\begin{abstract}
Resumen
Desde hace dos décadas, el análisis estratigráfico se viene aplicando a las fábricas arquitectónicas con el objetivo del conocimiento y la formulación de hipótesis de periodos constructivos. Este tipo de análisis representa además un momento de reflexión para el proyecto de restauración arquitectónica. El análisis estratigráfico de la arquitectura brinda un cuadro abundante de los datos materiales que pueden ser objeto de conservación o eliminación según el criterio del proyectista, basado en su sensibilidad, conocimiento, intención, etc. El análisis estratigráfico no proporciona ningún tipo de indicación sobre la modalidad del proyecto más adecuada a cada caso y no es garante de la conservación de la información estratigráfica. Efectivamente, la elaboración de un mismo análisis estratigráfico puede generar proyectos de restauración completamente diversos. El objetivo del texto consiste en plantear los temas y las opciones proyectuales que se abren tras de la realización de un análisis estratigráfico de la arquitectura.
\end{abstract}

Palabras clave: estratigrafía de la arquitectura, proyecto, restauración, conservación, estudios previos.

\begin{abstract}
Stratigraphic analysis is being applied to architectonic fabrics since two decades with the aim of researching them and propose a hypothesis of the constructive phases of the building. Besides, this type of analysis represents a period of reflection for the architectural project. Stratigraphic analysis of architecture offers a very complete idea of the material data that can be preserved or cancelled depending the architect's criteria. These criteria are based upon the architect's sensibility, knowledge, intention, will, etc. Stratigraphic analysis neither gives any indication or advice about the most convenient type of intervention nor guarantee the conservation of stratigraphic data. Indeed, the same stratigraphic analysis may generate very different and varied restoration projects. This paper tries to discuss about the questions and the several project options that lay behind the making of an architectural stratigraphic analysis.
\end{abstract}

Key words: Stratigraphy of architecture, project, restoration, conservation, prior studies.
"La obra debe continuamente "hablar" de sí misma y sólo con su propia voz, aunque las estratificaciones, las discontinuidades y las lagunas ofrezcan un mensaje incierto, conflictivo o contradictorio" (P. Torsello, 1988)

\section{EL ANÁLISIS ESTRATIGRÁFICO APLICADO A LA ARQUITECTURA}

El método del análisis estratigráfico, perteneciente a la disciplina de la arqueología, se viene aplicando desde hace dos décadas a la arquitectura (C. MileTO, 2000, p. 81). El análisis estratigráfico, que se aplica a las superficies de la arquitectura como única parte visible de los elementos arquitectónicos, consiste en una documentación de los datos materiales (materiales de construcción, técnicas constructivas, etc.) y en un análisis de las relaciones estratigráficas (de contemporaneidad y anterioridad/posterioridad) entre las Unidades Estratigráficas Constructivas (UEC). La aplicación del análisis estratigráfico a la arquitectura desvela el interés de historiadores y arqueólogos que pueden recoger la información que permite ampliar el conocimiento histórico del edificio, de las técnicas constructivas, de las tipologías arquitectónicas, etc. En este sentido, el objetivo principal de este tipo de análisis consiste en la formulación de una hipótesis de las fases y periodos constructivos del edificio o de las fábricas analizadas pero, al mismo tiempo, existe un objetivo secundario en la confección de una exhaustiva documentación de los materiales y técnicas constructivas en su relación estratigráfica y dentro de una cronología relativa, que permite la ampliación del conocimiento de la historia de la cultura material.

Además, el análisis estratigráfico, en el momento que se aplica a la arquitectura, suscita también interés en los arquitectos que se ocupan de la arquitectura histórica y de su restauración y conservación. En este sentido, este tipo de análisis de la arquitectura representa para el arquitecto una herramienta para su trabajo como estudio histórico material que proporciona datos sobre la historia del edificio. Los datos histórico-materiales identificados representan una parte del estudio que el arquitecto debe realizar antes de redactar un proyecto de restauración. El estudio previo (J. ESTEBAN CHAPAPRía, 1990, pp. 159-176) consiste en un conjunto de estudios de la mayor amplitud posible, a tenor del caso analizado, que permitan ampliar el conocimiento del edificio: estudios histórico-documentales, análisis estratigráficos, análisis de las patologías materiales y estructurales, estudios medioambientales, etc. Una vez recogida toda la información sobre el edificio, el proyectista puede proceder a redactar su proyecto en función de las necesidades identificadas (de conservación material, consolidación estructural, funcionalidad, etc.) y a las aspiraciones personales y de la sociedad (F. Doglioni, G. GiUni, 1997, p.14). 
Los datos recogidos en el análisis estratigráfico constituyen los documentos materiales que permiten el conocimiento de la historia del edificio pero, al mismo tiempo, componen la materia del edificio que el proyectista debe restaurar. La manipulación de la materia y, por tanto, de los datos estratigráficos por parte del arquitecto puede verificarse de diversas maneras según los criterios de proyecto que el mismo arquitecto establece y en función del valor que se atribuya a los mismos datos estratigráficos. De hecho, se les puede atribuir un valor documental que termina en el momento que se lee e interpreta el edificio o se pueden considerar como un valor añadido por su potencial ilimitado como documento susceptible de ampliaciones sucesivas en virtud de ulteriores estudios y puntos de observación diversos. En este caso, si se considera el dato material como un ilimitado potencial de información, la conservación del mismo se antoja indispensable para salvaguardar la posibilidad de una continua revisión e interpretación.

\section{POSIBLES CRITERIOS DE RESTAURACIÓN FRENTE A UN ESPACIO ESTRATIFICADO}

Sin embargo, la redacción de un análisis estratigráfico no garantiza por sí sola la conservación de la misma estratificación. A un mismo análisis estratigráfico puede seguir cualquier tipo de intervención en función de las expectativas que el proyectista se crea respecto al objetivo que pretende alcanzar: desde la reintegración de la imagen, la recuperación tipológica y la revalorización del espacio mediante una interpretación personal de los datos históricos y materiales; hasta la conservación de los datos materiales para proteger un conocimiento adquirido y para garantizar la posibilidad de nuevas lecturas a realizarse en un futuro. En cada caso se pueden identificar una serie de ventajas y desventajas ligadas a los criterios y a los resultados de la actuación.

\section{La homogeneización y la nueva configuración}

El objetivo de la homogeneización es la eliminación de la complejidad del espacio estratificado, además de las razones funcionales y distributivas. La ventaja de esta opción consiste en la funcionalidad que el edificio alcanza tras la intervención. Las desventajas son múltiples: desde la destrucción u ocultamiento de la materialidad, hasta la pérdida total de las posibles lecturas futuras, pasando por la pérdida de la conciencia del usuario de la historicidad del lugar.

\section{La recuperación selectiva}

Se trata de la revalorización del espacio materializada por una selección de la estratificación so color de criterios de recuperación formal o de recuperación tipológica. Los criterios de selección se amoldan a las situaciones específicas y pueden generar diferentes tipos de intervención:

\section{a) Recuperación de una configuración dominante o considerada prioritaria (fig. 1)}

En el caso de un espacio estratificado se puede plantear la recuperación de una configuración dominante o de una configuración juzgada más interesante según un criterio

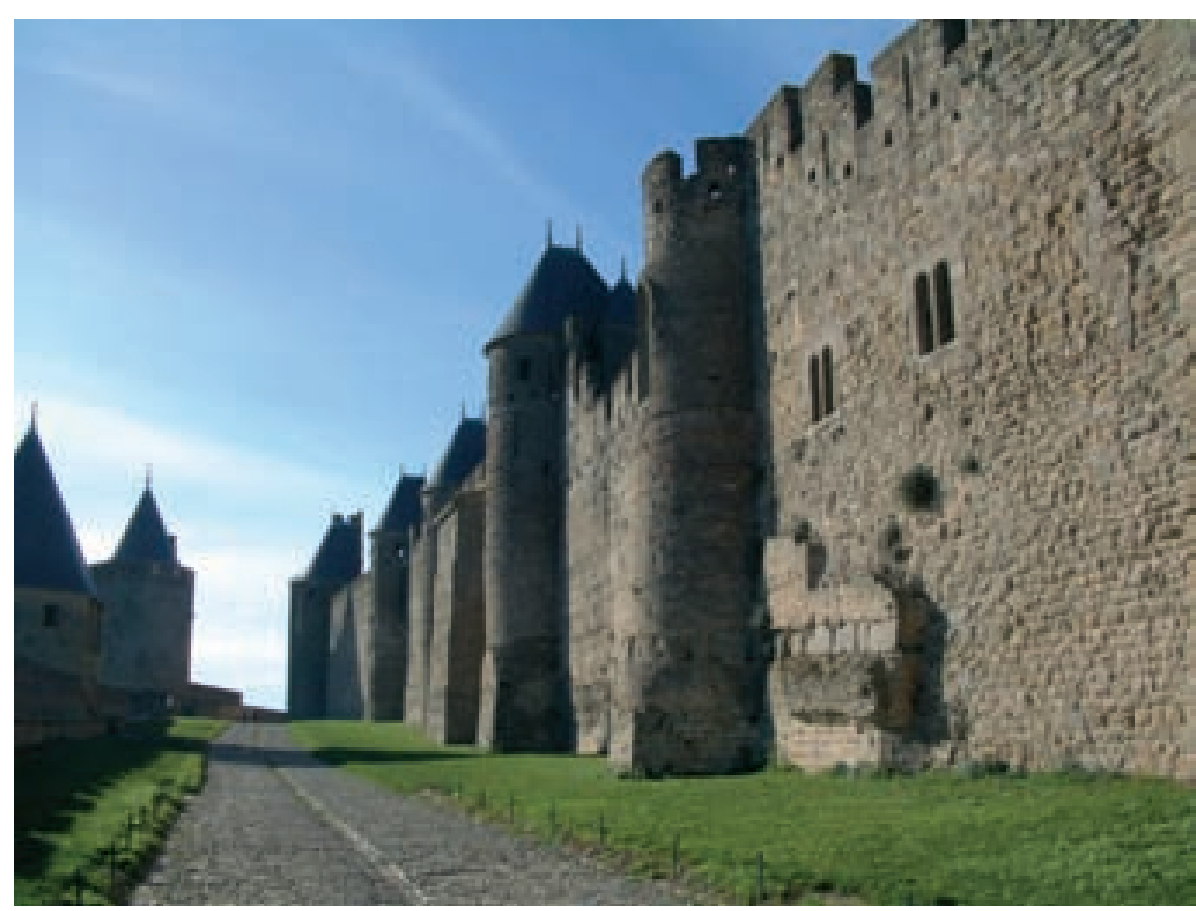

Fig. 1. La intervención de recuperación de la configuración dominante en la ciudad amurallada de Carcassonne 
Fig. 2. Intervención de remoción de la fase barroca de la iglesia gótica de San Pere en San Mateu (Castellón)

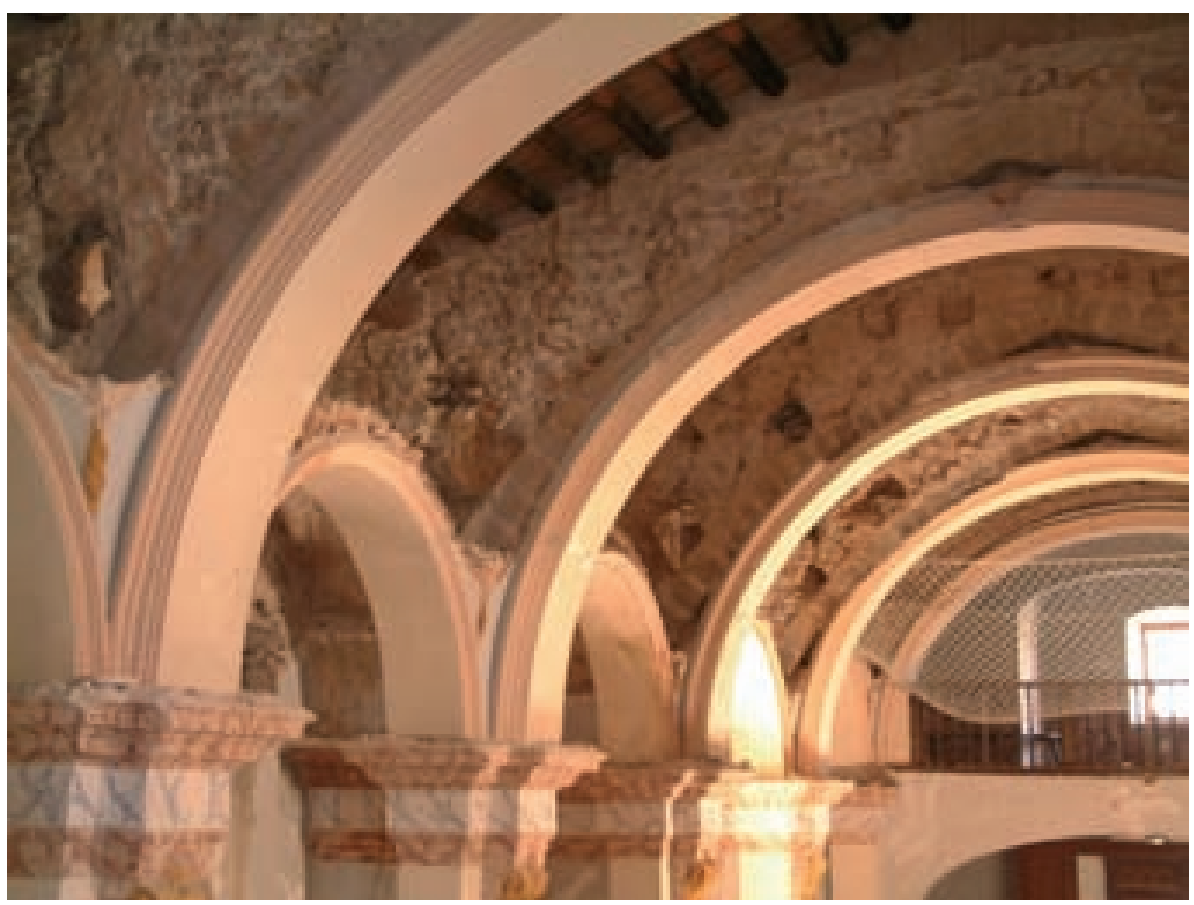

formal, histórico o tipológico. Esta intervención se lleva a cabo mediante la eliminación de todos los añadidos posteriores. La ventaja de este planteamiento reside en la recuperación de una imagen o tipología. Las desventajas radican en la pérdida de los datos materiales que no son congruentes con la configuración recuperada y en la eliminación de posibles futuras interpretaciones diferentes respecto a la impuesta por el juicio crítico del proyectista.

\section{b) Remoción selectiva de una configuración (fig. 2)}

Se trata del caso de edificios o espacios estratificados donde la estratificación se compone de dos o más configuraciones formalmente definidas y superpuestas. Se puede optar por la recuperación de la configuración anterior con la eliminación de la o las configuraciones sucesivas sobre la base de un juicio crítico de valor histórico o tipológico. La ventaja de ese planteamiento reside en la recuperación de unos datos históricos valiosos. Pero las desventajas son importantes: frente a la recuperación de una configuración se pierde otra configuración que presenta un valor histórico-documental; además se pierde la posibilidad de eventuales lecturas de la estratificación del edificio en el futuro.

\section{c) Inserción de fragmentos dentro de una nueva configuración o musealización (fig. 3)}

Se trata de una intervención donde se seleccionan sólo algunos fragmentos de la estratificación como resultado de un juicio crítico con objetivos didácticos, expositivos o compositivos. Las ventajas de esta opción residen en la conservación parcial de algunos fragmentos. Las desventajas residen en: la eliminación de gran parte de los datos; la eliminación de posibles lecturas futuras de la estratificación cerrada en una interpretación indiscutible por parte del proyectista; la eliminación completa del contexto de los fragmentos que se reducen a simples hallazgos incrustados en una pared o sueltos en un espacio completamente ajeno, fragmentos incapaces de contar su historia.

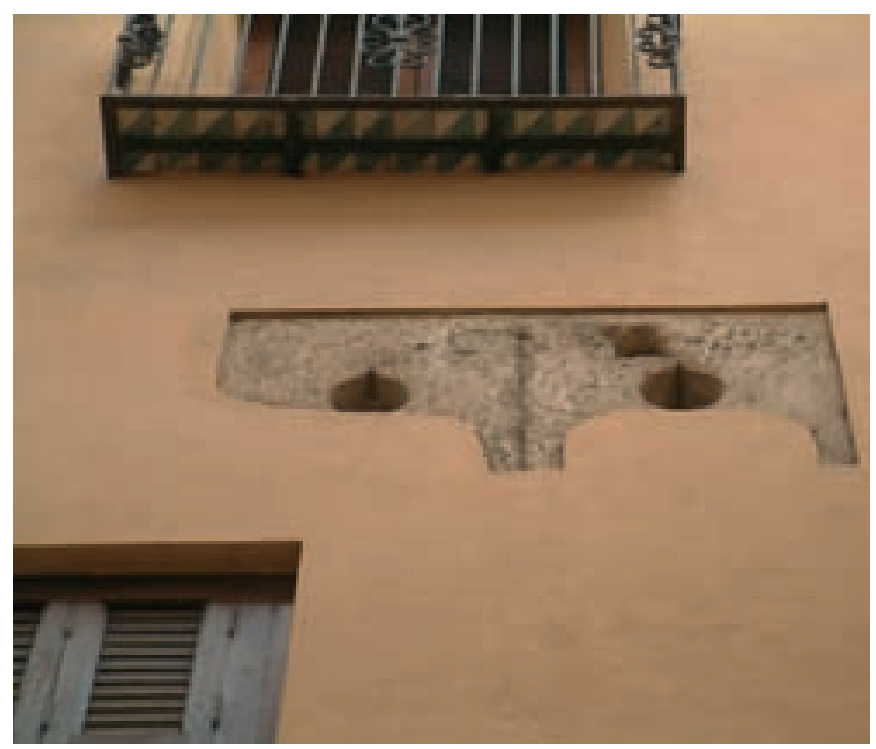

Fig. 3. Fragmento musealizado en la fachada de un palacio del centro histórico de Valencia 


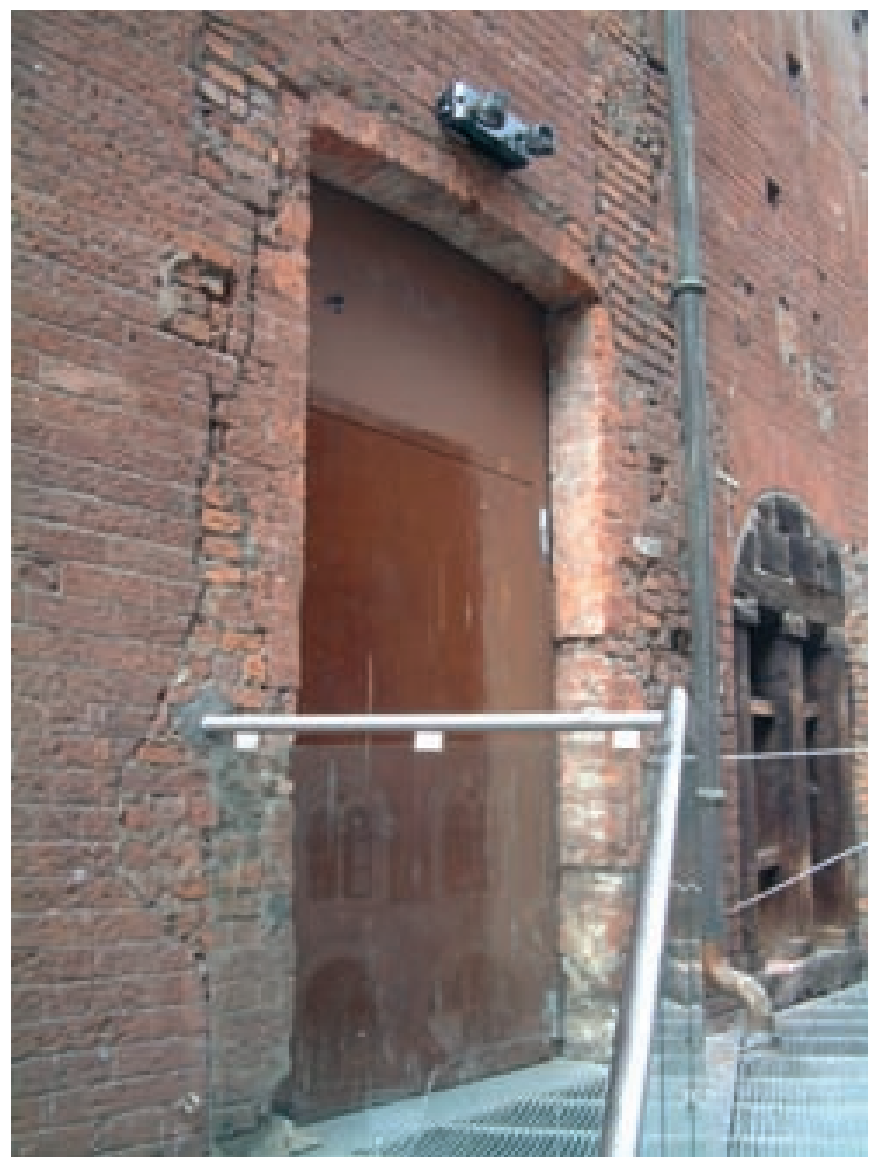

Fig. 4. Conservación integral de las huellas estratigráficas en el Palazzo della Ragione de Milán

\section{La conservación integral (fig.4)}

Los defensores de la conservación integral de la materialidad del edificio estratificado esgrimen esta opción como única intervención posible para conservar los datos y su lectura. Las ventajas de esta opción son: la conservación integral de los datos materiales y de la garantía de las posibles lecturas en el futuro. La desventaja más clara reside en el riesgo de una conservación indiscriminada de todas las huellas, desde las más reveladoras hasta las cicatrices banales originadas por la rutina constructiva reciente más convencional. Una dificultad de esta actitud reside en el contraste que se puede producir entre los paramentos antiguos y los nuevos elementos insertados con objetivos funcionales.

Una interpretación equivocada de los criterios y las intenciones de la opción de la conservación integral puede llevar a realizar dos tipos de intervenciones:

\section{a) Exposición de la plasticidad del patch-work (fig. 5)}

Se trata de intervenciones donde se aprovechan la variedad y la fragmentación de la estratificación como una excusa es-

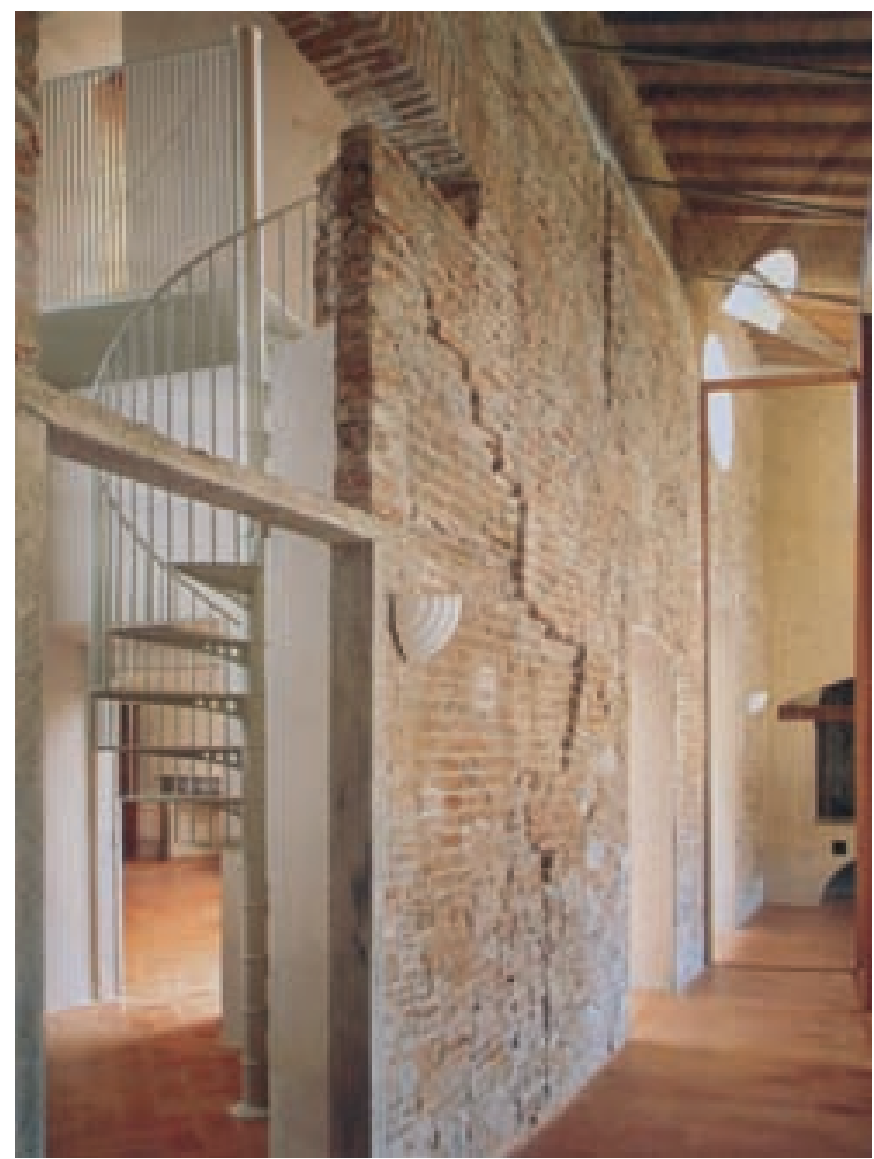

Fig. 5. Conservación estética del paramento estratificado en Casa Balbarini en Pisa

tética. La ventaja reside en la conservación de una gran cantidad de datos y en parte de la posibilidad de su lectura. Las desventajas son: el riesgo de fetichizar la estratificación mediante la manipulación conceptual de los elementos; y la tentación de desnudar cualquier paramento sólo con el objetivo de encontrar un fondo sugerente para la arquitectura contemporánea.

\section{b) Ostentación didáctica (fig.6)}

La otra faceta de la interpretación equivocada de los criterios de la conservación de los datos consiste en la realización de una intervención dirigida a explicar ostensiblemente la interpretación de la estratificación. La ventaja de este tipo de intervención sería la aparente conservación de los datos. Pero la desventaja principal consiste en el congelamiento de la estratificación en una determinada hipótesis que no dejaría espacio a otras posibles interpretaciones futuras. La aparente conservación integral se transformaría de esta manera en la momificación de un palimpsesto, que quedaría congelado en la ostentación de un conocimiento. 


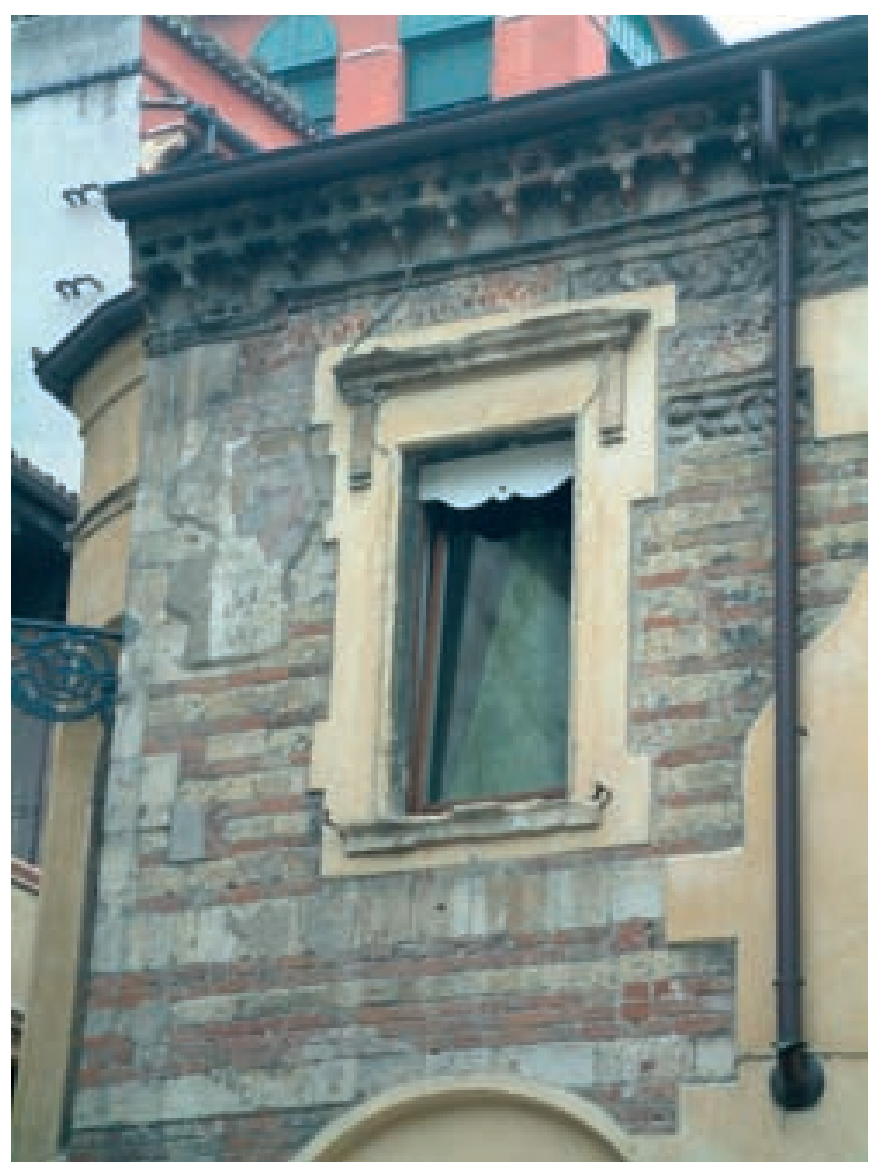

Fig. 6. Ostentación de la estratificación en la fachada de la iglesia de San Giovanni in Foro en Verona

\section{REFLEXIONES PARA LA CONSERVACIÓN DE LA ESTRATIFICACIÓN ARQUITECTÓNICA}

Según la línea de conservación que los autores de este texto están llevando a cabo desde hace unos años, difícilmente se podría abrazar plenamente una u otra opción precedentemente descritas. Sin embargo en cada una de ellas existen aspectos aislados que pueden servir para la elaboración de una nueva alternativa de proyecto (MiLETo C., 2004). La conservación de la estratificación arquitectónica, según la línea que se propone, no se agota en la conservación de los datos materiales, sino requiere que el proyectista centre su atención también en las componentes de comunicación y vivencia de la arquitectura. Por tanto, la conservación de la estratificación arquitectónica reside en tres elementos diferentes pero no excluyentes.

En primer lugar, la conservación de los datos materiales (materiales y técnicas constructivas) y de la posibilidad de un análisis futuro. Garantizar simplemente la permanencia de los fragmentos congelaría la estratificación en la interpretación actual. Es necesario garantizar la posibilidad de lecturas futuras para conservar realmente la estratifica- ción. Este aspecto de la conservación se alcanza mediante la conservación material de los fragmentos (UEC) y de las huellas estratigráficas que permiten la lectura de las modalidades de transformación (bordes entre unidades, es decir la junta entre las diferentes UEC). La conservación de los datos materiales se basa en el necesario conocimiento por parte del arquitecto de los mecanismos de estratificación que permite proyectar la propia intervención mentalidad estratigráfica como una fase más de la estratificación (F. DOGLIONI, 1997, pp. 288-289).

En segundo lugar, la conservación del carácter estratificado de la arquitectura. La arquitectura estratificada se presenta: heterogénea, es decir, compuesta por fragmentos diferentes entre ellos en textura, colores, técnicas, etc.; fragmentada, o sea, compuesta por elementos no concluidos que remiten a totalidades desaparecidas. La presencia de estos fragmentos remite a la ausencia de espacios antiguos. Los fragmentos constituyen la memoria de una historia personal del edificio y de una historia colectiva; y temporalizada, es decir, una arquitectura que con su configuración actual remite al paso del tiempo. Es el tiempo de las generaciones que se suceden transformando el edificio y es el tiempo de la materia que se transforma lentamente y que con su disgregación permite entrever las fases constructivas anteriores. La conservación de las tres características de la arquitectura estratificada es necesaria para la conservación de su carácter.

En tercer lugar, la conservación de la experiencia del fruidor frente a una estratificación arquitectónica. La experiencia consiste en: la percepción de la materialidad, es decir, colores, texturas, formas, elementos llamativos, etc.; la experiencia de gusto ligada a la experiencia de la complejidad del espacio y a su legibilidad; y el conocimiento sensible ligado a la experiencia sentimental de la historia, del paso del tiempo y de la memoria. La conservación de estos tres aspectos es necesaria para la conservación de la experiencia de la estratificación arquitectónica.

Estas expectativas de conservación (conservación de la materialidad, del carácter y de la experiencia) deberán evidentemente compatibilizarse con las exigencias de permanencia (material, estructural, etc.) y de vivencia (funcionalidad y decoro).

\section{UN EJEMPLO DE PROYECTO DE RESTAURACIÓN ARQUITECTÓNICA}

\section{La fase previa de análisis estratigráfico}

Durante los últimos años, los autores de este artículo han debido reflexionar y afrontar de manera directa los problemas anteriormente expuestos, especialmente en un estudio y sucesivo proyecto de restauración de una sala interna del 
conjunto arquitectónico de la Alhambra, realizados por encargo del Patronato que rige el monumento.

Los muros perimetrales presentaban una estratificación de gran complejidad. Tras la elaboración del análisis estratigráfico constructivo, se detectaron cinco periodos principales en su configuración actual, que se ordenaron según una cronología relativa. Una vez contrastados estos datos con los documentos históricos, estos cinco periodos se identificaron con los siguientes lapsos de tiempo: el primer periodo, subdividido en dos fases, desde mediados del siglo XIV hasta 1492, fecha de la reconquista de Granada; el segundo periodo, también dividido en dos fases, desde 1492 hasta 1528, fecha de confección del plano de la Alhambra de Machuca; el tercer periodo, sin apenas documentos históricos de apoyo referentes a esta sala, desde 1528 hasta 1923, un prolongado periodo que se ha dividido en cuatro fases; el cuarto periodo, dividido en dos fases, desde 1923 hasta 1936, es decir, correspondiente a las actuaciones de Leopoldo Torres Balbás en la Alhambra; y el quinto periodo, desde 1936 hasta nuestros días.

\section{Los ámbitos arquitectónicos}

Esta hipótesis de periodos constructivos, además de proporcionar un conocimiento de la posible evolución del espacio en el tiempo, asume un rol de gran importancia en el análisis de los ámbitos arquitectónicos que se han ido superponiendo en el mismo espacio. Al mismo tiempo, este tipo de lectura nos permite considerar la unidad estratigráfica no sólo como elemento por sí mismo o testimonio material, sino como parte o testimonio de un determinado espacio histórico. En este sentido, el proyecto de restauración posterior del espacio debería tener en cuenta no sólo la unidad estratigráfica en sí misma sino el conjunto de unidades que caracteriza la huella de un determinado ámbito arquitectónico.

Para este estudio, se ha partido de la hipótesis de periodos, fases constructivas y unidades homogéneas de obra elaborada previamente a partir del análisis estratigráfico constructivo. Cada plano de ámbito arquitectónico refleja las fases de un determinado periodo correspondientes a dicho momento de la evolución del espacio de la sala más todas las unidades pertenecientes a los periodos precedentes. Se ha detectado la existencia de siete ámbitos diferentes correspondientes a los cinco periodos constructivos definidos previamente.

\section{Observaciones específicas}

Se han realizado una serie de observaciones específicas para la restauración de esta sala, que son independientes de la estratigrafía y se pasan a describir. En primer lugar, se deben distinguir las superficies según su voluntad de duración, de modo que existen superficies de acabado que requieren una renovación periódica (por ejemplo los encalados periódicos de los paramentos exteriores) o superficies que fueron pensadas como acabados definitivos (por ejemplo estucos interiores o paramentos de sillería o fábricas de ladrillo perfectamente aparejado). Por consiguiente, se trata de observar las características de acabado de las superficies: superficies creadas para permanecer a la vista frente a superficies nacidas para ser recubiertas, superficies pensadas como renovables frente a superficies de acabado definitivo.

En segundo lugar, la degradación de las superficies puede ser tan acentuada que obligue a una intervención necesaria de consolidación. Cabría distinguir otro factor entonces que discerniera las superficies muy degradadas de las superficies bien conservadas.

En tercer lugar, otro factor de carácter perceptivo a tener en cuenta, no por ello soslayable, es la diferente relación con la superficie del paramento en función de su altura relativa respecto a la vista humana. Así, se pueden distinguir tres bandas horizontales de influencia: inferior o zócalo, central o entorno y superior o friso. La ubicación inmediata de la zona de entorno respecto de la vista humana requiere de una atención especial en el tratamiento. Por otro lado, la ubicación mediata de la zona zócalo o la zona friso respecto de la vista humana, permiten un tratamiento más libre, rugoso o irregular.

Por último, cabría distinguir entre los conceptos de ámbito y espacio. La palabra espacio indica la organización tridimensional de los elementos que constituyen un lugar y posee connotaciones relacionadas con la geometría y el volumen. En cambio, la noción de ámbito recoge el contenido semántico de espacio y lo enriquece y amplía al incorporar también cualidades sensibles como la atmósfera, la luz, el color, la materialidad y la textura de los muros que conforman ese ámbito. La propuesta de proyecto no debería perseguir tanto la recuperación del espacio de antaño, entendido como volumetría, sino más bien la evocación de los ámbitos a través del mantenimiento en la medida de lo posible y lo razonable de las cualidades físicas de los muros que lo conforman.

\section{Descripción de las diversas opciones de intervención}

Para la toma de decisiones del proyecto, partiendo de diferentes criterios, se realizaron seis propuestas de intervención cuyo impacto en la realidad se ha estudiado gráficamente, con la descripción de las actuaciones previstas en cada caso y el conjunto de ventajas e inconvenientes que reúne cada intervención, expuesto en modo que se pueda 


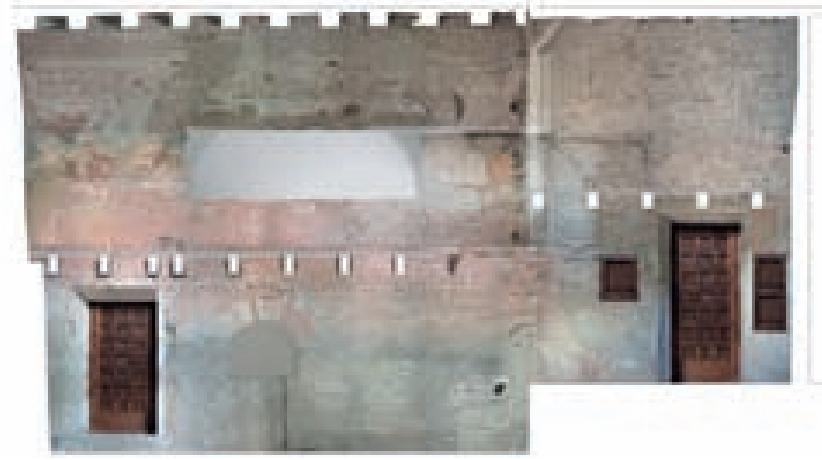

Fig. 7. Propuesta de conservación y consolidación del estado actual

ponderar la bondad de cada una de las opciones. Las intervenciones se han ordenado de la más inocua con los muros existentes, que respeta todas las huellas existentes (propuesta 1), hasta la más sumaria en el tratamiento de los mismos, que cancela dichas huellas en aras de una homogeneización del tratamiento interior de los espacios (propuesta 6). Todas estas opciones varían únicamente en el tratamiento de las superficies, y comparten una misma distribución en planta e idéntico tratamiento de los huecos existentes, cuya configuración se ha justificado en los capítulos anteriores.

En la primera propuesta (conservación y consolidación del estado actual según las modalidades de una conservación integral) se prevé la limpieza de los muros, la consolidación de los mismos con consolidantes transparentes (agua de cal, silicato de etilo...) y la fijación de los enlucidos existentes. La ventaja reside en la conservación integral de los datos y su lectura. La desventaja principal consiste en la conservación indiferenciada de cualquier tipo de huella.

Siguen tres propuestas de conservación selectivas sobre diferentes bases conceptuales. En las tres la ventaja radica en la conservación de los datos materiales, algunos

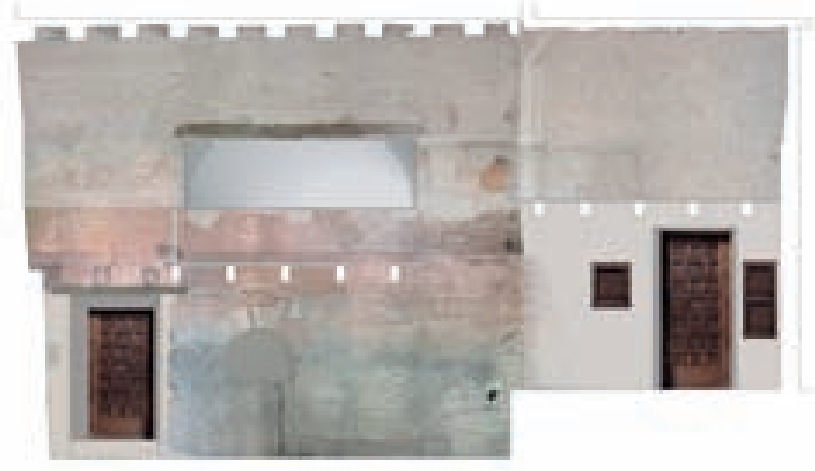

Fig. 9. Propuesta de diferenciación por ámbitos históricos y arquitectónicos

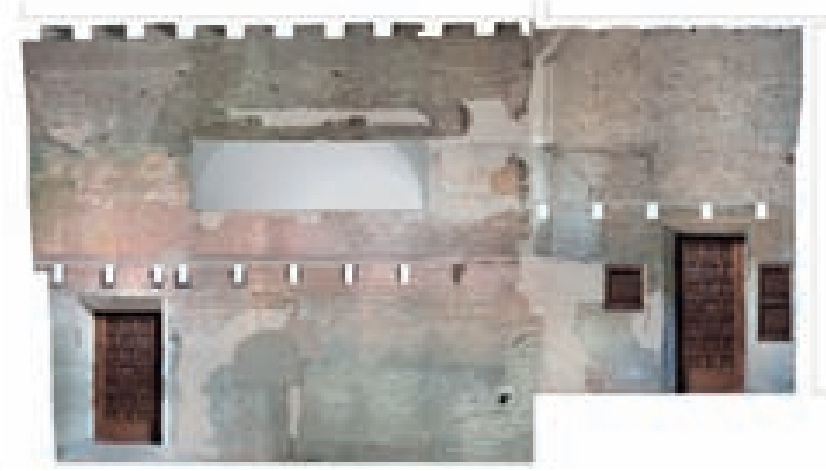

Fig. 8. Propuesta de consolidación y cubrición de las partes degradadas

visibles y otros ligeramente velados. La desventaja reside en la pérdida parcial de la lectura de la estratificación. Una de ellas (propuesta 2) consiste en la consolidación del estado actual y cubrición de las superficies degradadas con una lechada de cal. Previa limpieza de los muros existentes, se propone una consolidación de los mismos y, únicamente en el caso de las superficies degradadas, la aplicación de una lechada de cal que repare las mismas y les confiera un aspecto más decoroso a través de un revestimiento translúcido uniforme. Otra opción (propuesta 3) consiste en la diferenciación por ámbitos históricos y espaciales mediante la aplicación selectiva de aguas, lechadas y enlucidos de cal. Se trata de la combinación de los tratamientos de limpieza, consolidación, aplicación de lechadas y enlucidos de cal sobre las superficies de una manera selectiva y crítica, que persiga la diferenciación de los ámbitos. Y la siguiente (propuesta 4) consiste en el revestimiento de aquellas superficies que históricamente estaban revestidas. Se trata de revestir con un enlucido de cal de manera coherente con su disposición original, todas aquellas superficies concebidas en su día como revestidas, con independencia de otras consideraciones de tipo histórico-constructivo.

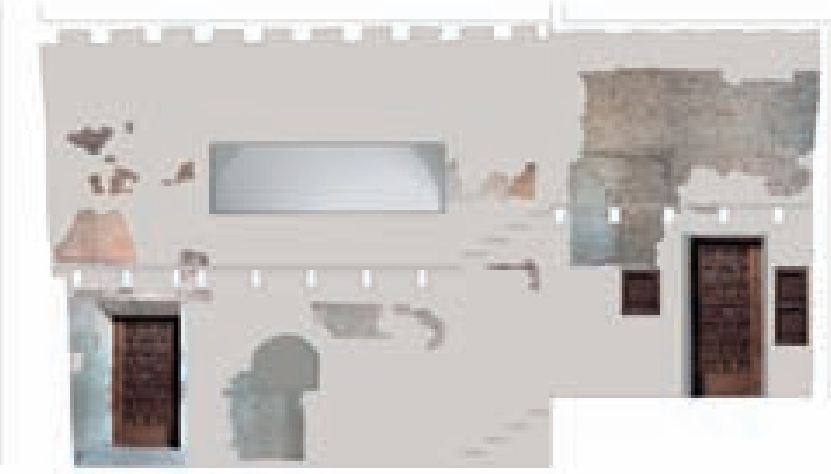

Fig. 10. Propuesta de revestimiento de las superficies históricamente revestidas 


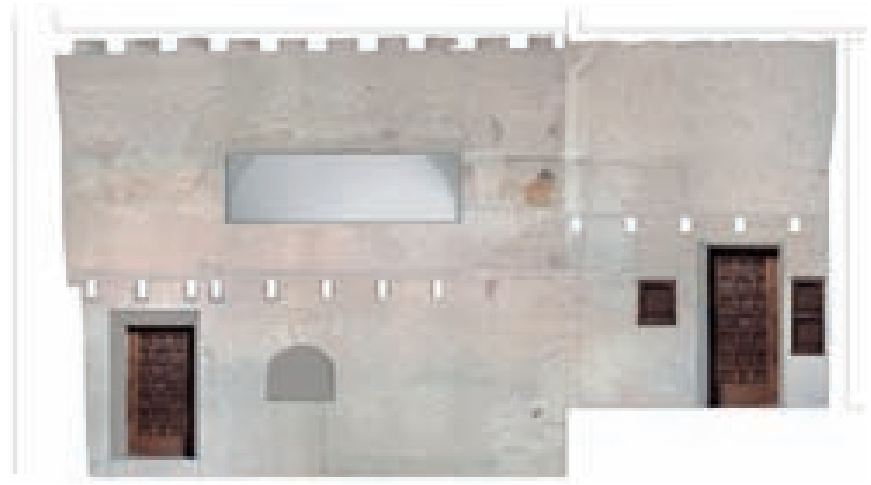

Fig. 11. Propuesta de aplicación de una veladura de lechada de cal sobre el conjunto de las superficies

Por último, siguen dos diferentes propuestas de homologación de las superficies. La ventaja reside en la eliminación de la simplicidad del espacio. La desventaja reside en la pérdida de los datos, de su lectura y de la percepción de la historicidad del lugar. Una de estas alternativas (propuesta 5) consiste en la aplicación de una veladura de lechada de cal sobre el conjunto de las superficies, con independencia de su lógica histórico-constructiva y su estado precedente visto/enlucido. Y la última opción (propuesta 6) consiste en el revestimiento opaco y uniforme con enlucido de cal de todo el interior del espacio, ignorando todas las huellas y datos estratigráficos y materiales.

Entre todas la opciones barajadas se considera que la propuesta 3 ofrece un buen compromiso entre las expectativas de conservación (conservación material, del carácter y de la experiencia) y las necesidades de permanencia (estructurales, materiales, etc.) y de vivencia (funcionalidad y decoro).

\section{CONCLUSIÓN}

Con las ideas propuestas en este breve texto se ha pretendido evidenciar la necesidad de una reflexión profunda y un análisis razonado de las diversas opciones de intervención en el momento de redactar un proyecto de restauración sobre una arquitectura estratificada. Es innegable la enorme responsabilidad del arquitecto restaurador en el momento de proyectar y realizar una obra de restauración sobre una

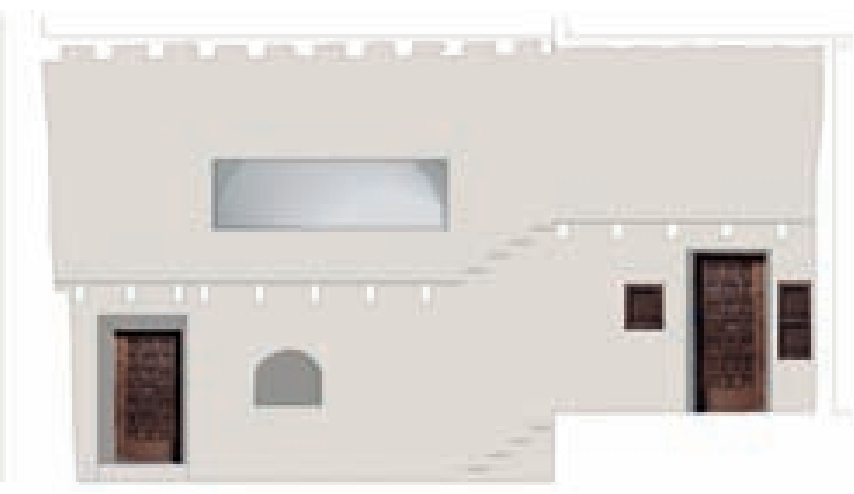

Fig. 12. Propuesta de revestimiento opaco con enlucido de cal de todas las superficies

arquitectura estratificada que, además de constituir un conjunto de datos históricos documentales auténticos y únicos, representa una arquitectura que transmite mensajes gracias a sus características físicas y a la percepción de las mismas. El proyectista, por tanto, además de aguzar los sentidos frente al carácter heterogéneo, fragmentado, y temporalizado de la arquitectura estratificada, debería aprender a entender y apreciar el lenguaje de la estratificación no sólo para poderla analizar, tarea que en gran parte ya realizan otros especialistas (historiadores y arqueólogos), sino sobre todo para llegar a estimarla en un grado suficiente que le permita proyectar una intervención cuidadosa y respetuosa de los datos materiales y de sus significados inmateriales.

\section{Bibliografía}

Doglioni, F., Giuni, G., Progettare il restauro. Esperienze didattiche nello IUAV. Catalogo della mostra, IUAV, Venecia, 1997

Doglioni, F., Stratigrafia e restauro, Lint, Trieste, 1997

ESTEBAN CHAPAPRía, J., Estudio previo a la intervención en el Patrimonio Arquitectónico, en Monumentos y Proyecto. Jornadas sobre criterios de intervención en el patrimonio arquitectónico, Ministero de Cultura, Madrid, 1990, págs. 159-176

MiLETo, C., Algunas reflexiones sobre el Análisis Estratigráfico Murario, en Loggia, n. ${ }^{9}$ 9, Valencia, 2000, págs. 80-93

MiLETO, C., La estratificación arquitectónica. Análisis, experiencia y conservación de las huellas de la historia, tesis doctoral inédita, Universidad Politécnica de Valencia, 2004, directores: J. F. Noguera Giménez y F. Vegas López-Manzanares. 